\title{
The Infineon Silicon MEMS Microphone
}

\author{
Alfons Dehé, Martin Wurzer, Marc Füldner and Ulrich Krumbein \\ Infineon Technologies AG, 81726 Munich, Germany \\ alfons.dehe@infineon.com
}

\begin{abstract}
This paper reports on the state of the art silicon micromachined microphone utilizing a dual poly silicon membrane system. MEMS chips from $1.4 \mathrm{~mm}$ down to $1.0 \mathrm{~mm}$ side length are applied for mobile communication. Design aspects related with key performance parameters such as sensitivity, signal to noise ration and distortion are discussed. Sensitivity of $-38 B V / P a$ is achieved for different microphone membrane diameters. A maximum signal to noise ration of $66 \mathrm{~dB}(\mathrm{~A})$ for the largest system could be achieved. The perfect fit of simulation versus measurements enables deeper analysis and balancing of noise contributors. Environmental noise suppression of $5 \mathrm{~dB}$ by acoustical high pass design is demonstrated.
\end{abstract}

Key words: microphone, silicon MEMS, membrane, signal to noise ratio, noise suppression.

\section{Introduction}

Today silicon micromachined microphones have reached a mature status of production after a long history of development [1] and market introduction into mobile phones. With the ever increasing shares of smart phones with up to three microphones for voice pick-up, hands free and noise suppression applications the annually produced volumes tend towards several billion pieces per year. Being successful in such a market requires proper design and modeling of the system performance as well as a stable semiconductor manufacturing processes. The requirements of microphones for mobile applications have significantly increased during the last years from acceptable telephone quality with signal to noise ratio of $58 \mathrm{~dB}(\mathrm{~A})$ up to a state of the art SNR of $66 \mathrm{~dB}(A)$. The desire to record music with ultimate dynamic range of more than $110 \mathrm{~dB}$ still pushes for lower self noise and higher linearity. Following this roadmap the applications of the early development days such as hearing aids or automotive hands free supplies merge with the advanced mobile specifications [2].

In the first section this paper describes the construction and technology details of the acoustical transducer. Following discussion of key performance parameters gives insight into the miniaturization challenge. A discussion of the pressure ventilation at low frequencies explains how effective environmental noise can be filtered. Finally, module measurements are compared to simulations allowing the detailed noise source analysis.

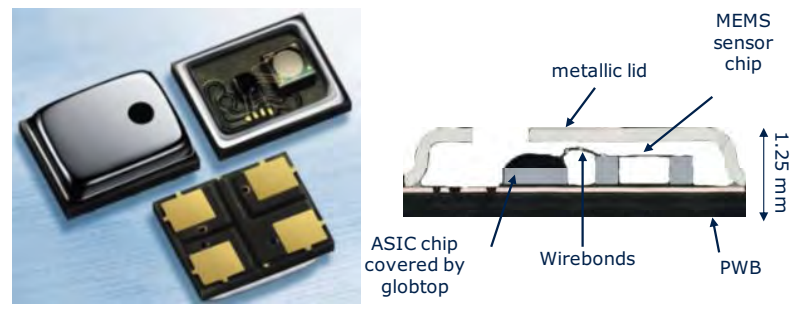

Fig. 1. A silicon microphone top sound port module on the left with and without metal cap and in a cross sectional cut displaying the ASIC and the MEMS chip with the chip cavity acting as back volume.

\section{Design and Technology}

The microphone follows the condenser principle: the sound enters the housing through a sound port and drives a movable membrane against an enclosed back volume (fig. 1). The membrane moves according to amplitude and frequency of the impinging sound. To pick up an electrical signal the membrane represents one electrode of the biased condenser and the other one is represented by a perforated and rigid so called backplate. The constant charge biasing and read out of voltage change according to membrane displacement is done by an ASIC that finally delivers an analog or digital output signal. The housing of the microphone protects the device, provides EMI shielding and is part of the acoustical design. 


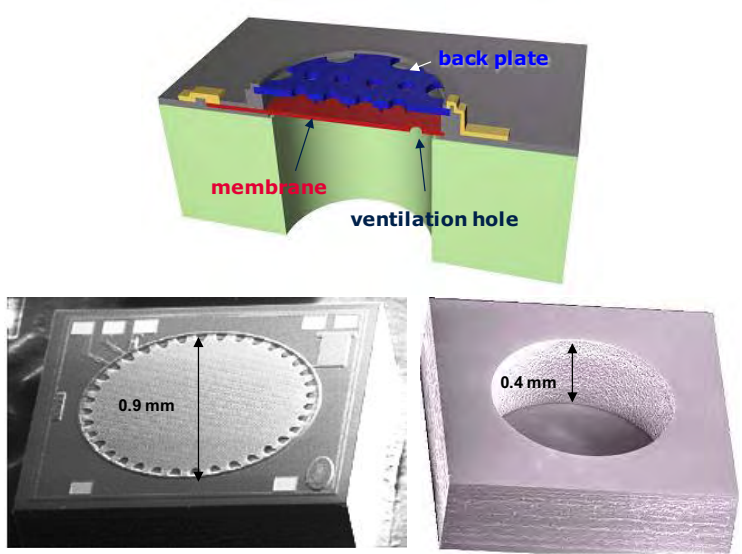

Fig. 2. Schematic and MEMS chip from front and backside.

Figure 2 shows a schematic cross-section as well top and bottom views of a MEMS chip. In more detail the microphone has a circular poly silicon membrane of 0.7 to $1.1 \mathrm{~mm}$ diameter depending on design variant.

The close up picture (fig. 3) shows the MEMS stack prior to the final oxide etch that is applied in the fabrication to release the membrane. The $330 \mathrm{~nm}$ thin poly silicon membrane is spanned over the back cavity with a well controlled tensile stress. An air gap is defined by a $2.2 \mu \mathrm{m}$ thick oxide layer. And a top backplate is constructed from a composite of highly tensile silicon nitride and thick poly silicon layer. It gives enough rigidity to act as a reference electrode.

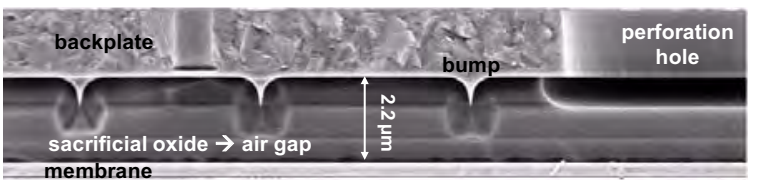

Fig. 3. Cross-section of the MEMS stack prior to release etch.

The perforation holes inside the backplate play an important role in transmission of sound towards the membrane. Diameter and density of holes (fig. 4) are the key parameters for noise contribution. Furthermore the backplate exhibits tiny needle type structures that prevent the sticktion of membrane and backpalte after wet sacrificial etching and drying. They also introduce robustness under humidity or even direct wetting.

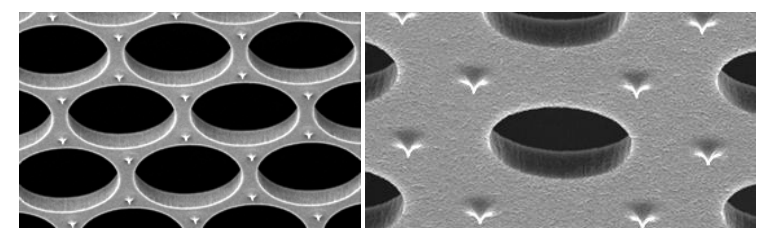

Fig. 4. Backplates with different perforation densities and anti sticking bumps facing the membrane.
Figure 5 gives a close up of the edge region of membrane and backplate resting on the rim of the dry etched cavity. Small holes inside the membrane layer allow ventilation of pressure between front and backvolume of the microphone. They form an acoustical high pass for sound.

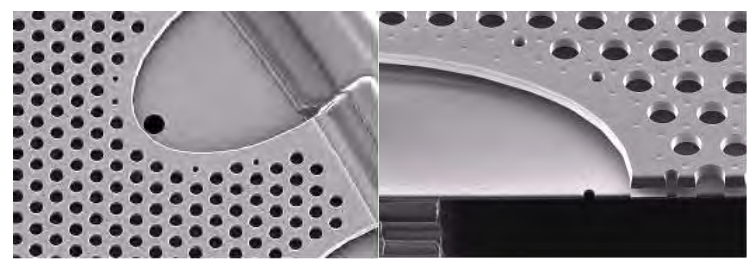

Fig. 5. Insight of the backplate and membrane showing the perforation hole (FIB cut on the right side).

\section{Key Performance Parameters and Design}

Despite the sensitivity of the membrane the key performance indicator is the noise level typically weighted by the human ears $A$ filter characteristic [3]. From a simplified equivalent lumped elements model for small signals the basic influence parameters can be understood (fig. 6). The sensitivity of the system is a combination of compliance of membrane $\mathrm{C}_{\text {mem }}$ and encapsulated air volume $\mathrm{C}_{\mathrm{bv}}$. The noise is dominated by the resistive components of air streaming through the perforated backplate $R_{\text {perf, }}$ the ventilation hole $R_{\text {vent }}$ and the sound port $R_{\text {port }}$. Low harmonic distortion at high sound pressure levels is must as well. The biasing and read out of the capacitive sensor with the help of an ASIC is adding amplification, noise and distortion. Those effects have to be controlled carefully but are not discussed in this article.
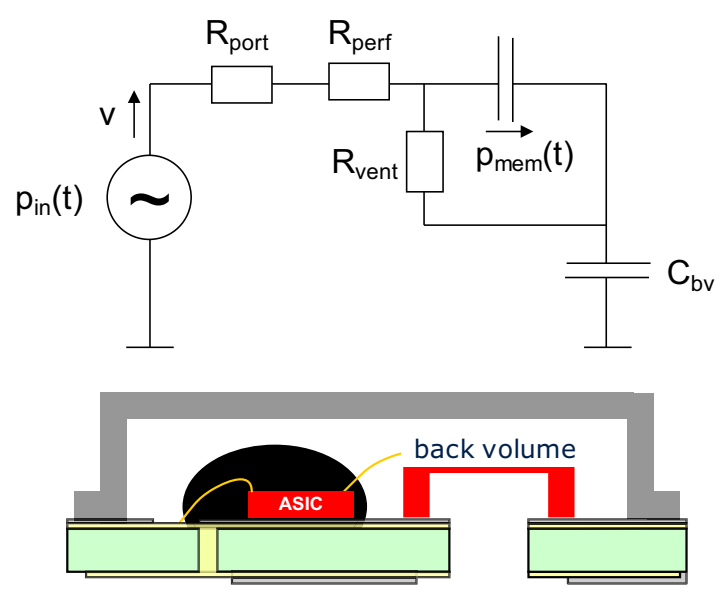

Fig. 6. Typical application of MEMS and ASIC in a housing with bottom sound port. The respective equivalent circuit is describing the critical parameters that can be influenced by design. 


\section{Membrane Design}

The sensitivity of the microphone is proportional to the compliance of the membrane. Despite the material parameters the design is an important parameter. Though spring supported designs have been proposed in the past [3] a circular clamped membrane is most robust with respect to stress concentration under extreme pressure conditions. If for a given diameter a more sensitive membrane is needed without change of material parameters we found the best choice is a corrugated membrane (fig. 7). Other than in the simplified schematic the real corrugation has smooth transitions to avoid stress concentrations. By number and height of the corrugation the final membrane compliance can be adjusted precisely.

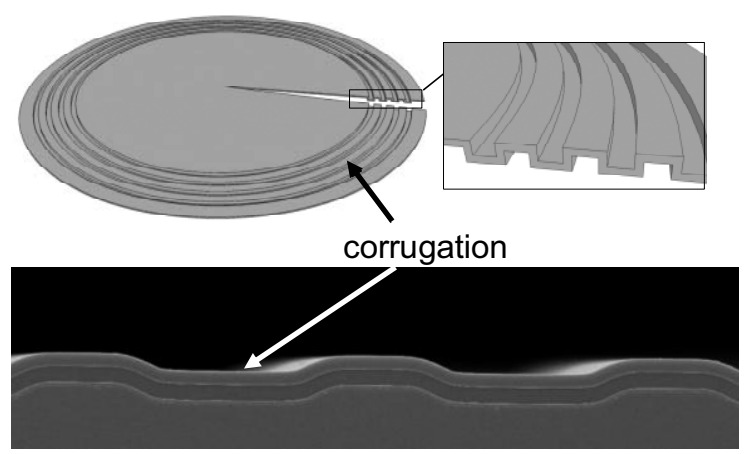

Fig. 7. Schematic of circular membrane with corrugation lines and technology cross section with the membrane layer in the cause of the fabrication process.

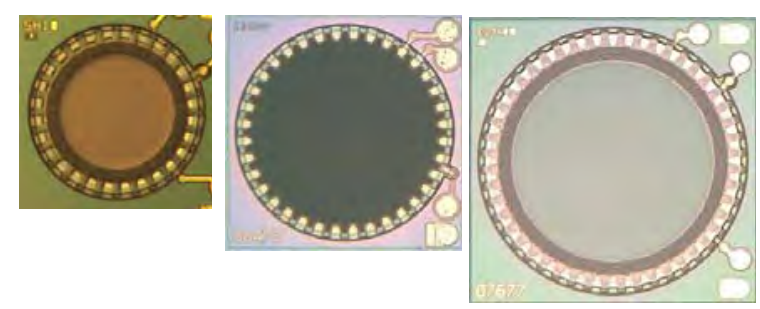

Fig. 8. Microphone chips with different membrane diameters $(0.7 \mathrm{~mm}, 0.9 \mathrm{~mm}$ and $1.1 \mathrm{~mm}$ left to right)

Table 1 gives an overview on sensitivity with respect to different membrane diameters according to figure 8 . Shrinking the chip size is demanded following commercial arguments but also to enable ever smaller footprints of the module packages. The target is to keep the system sensitivity constant but gain as much as possible signal to noise ratio. The membrane compliance is adjusted to have a constant value of $10 \mathrm{~nm} / \mathrm{Pa}$. This can be done by tuning the poly silicon membrane stress. The smaller the membrane the smaller is the active capacitance of the condenser and its relation to the parasitic capacitance plus the input capacitance of the amplifier is decreasing. The smallest membrane causes a signal loss of $4 \mathrm{~dB}$ due to this effect. The size of the back volume in the package is influencing the sensitivity of the microphone system. Reducing the backvolume from $3 \mathrm{~mm}^{3}$ to $2 \mathrm{~mm}^{3}$ is equivalent to a sensitivity loss of about $1 \mathrm{~dB}$. Consequently, the amplifier of the smallest system has a $6 \mathrm{~dB}$ amplification to compensate for those losses.

As a rule of thumb the noise contribution of the MEMS is nearly inverse proportional to the membrane area. Since the ASIC noise contribution stays constant the effect is smaller on system level as can be seen in table 1.

Tab. 1: Parameters and performance of state-ofthe-art MEMS microphone chips in the application.

\begin{tabular}{|c|c|c|c|c|}
\hline & Small & Medium & Large & \\
\hline Chip Area & $(1.0)^{2}$ & $(1.2)^{2}$ & $(1.4)^{2}$ & $\mathrm{~mm}^{2}$ \\
\hline Membrane Diameter & 0.7 & 0.9 & 1.1 & $\mathrm{~mm}$ \\
\hline Corrugation & \multicolumn{3}{|c|}{6} & \\
\hline Compliance & \multicolumn{3}{|c|}{10} & $\mathrm{~nm} / \mathrm{Pa}$ \\
\hline Back Volume & $\sim 1$ & $\sim 3$ & $\sim 3$ & $\mathrm{~mm}^{3}$ \\
\hline Corner Frequency & \multicolumn{3}{|c|}{80} & $\mathrm{~Hz}$ \\
\hline $\begin{array}{l}\text { active/parasitic } \\
\text { capacitance incl. } \\
\text { ASIC }\end{array}$ & $\sim 1$ & $\sim 1.6$ & $\sim 1.8$ & \\
\hline Gain & 6 & 0 & 0 & $\mathrm{~dB}$ \\
\hline Sensitivity & \multicolumn{3}{|c|}{-38} & $\mathrm{dBV} / \mathrm{Pa}$ \\
\hline $\begin{array}{l}\text { Signal to Noise } \\
\text { Ration }\end{array}$ & 61 & 64 & 66 & $\mathrm{~dB}(\mathrm{~A})$ \\
\hline
\end{tabular}

\section{Pressure Ventilation}

The effect of ventilation holes in the membrane is discussed with respect to different number $\mathrm{N}$ and diameter $D$. In analogy to an electrical highpass filter, the lower corner frequency $f_{\text {low }}$ can be expressed by

$$
f_{\text {low }}=\frac{1}{2 \cdot \pi \cdot R_{\text {vent }} \cdot C_{b v}}
$$

$R_{\text {vent }}$ is the equivalent acoustical resistance of the ventilation holes in the membrane [4]. The acoustical compliance of the back volume $C_{b v}=V_{b v} / \beta$ is given by the package volume $V_{b v}$ and the compressibility of air $\beta$.

In good agreement with the expectation the lower corner frequency increases with larger and more ventilation holes due to a smaller acoustical resistance $R_{\text {vent }}$ (tab. 2). Also, the maximum sound pressure level of an $80 \mathrm{~Hz}$ acoustic distortion source is stated in table 2 . Thereby, the maximum sound pressure of this distorter is defined by the microphone output of a regular signal of $1 \mathrm{kHz}$ and $1 \mathrm{~Pa}$ being compressed by more than $1 \mathrm{~dB}$ due to overloading of the amplifier. The $80 \mathrm{~Hz}$ test signal is a representative for external acoustical interferences at comparable low frequencies but high sound pressure levels.

As expected, the maximum allowed sound pressure of an $80 \mathrm{~Hz}$ acoustic interference is 
strongly correlated with the sensitivity attenuation at $80 \mathrm{~Hz}$ as shown in figure 9. The acoustical overloading of the amplifier occurs at higher sound pressure levels for higher corner frequencies. For suggested lower frequencies of external noise sources, the design of a membrane with a sufficient lower corner becomes more important.

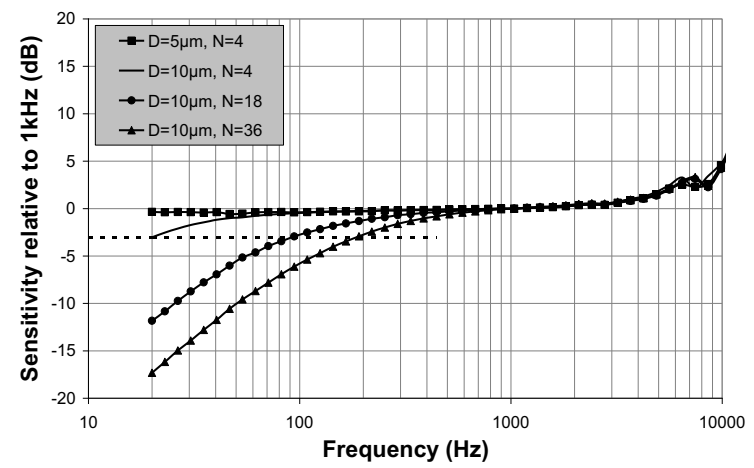

Fig. 9 Sensitivity over frequency relative to the sensitivity at $1 \mathrm{kHz}$ for different ventilation hole designs.

Tab. 2: Lower corner frequency and maximum sound pressure level (SPL) of an $80 \mathrm{~Hz}$ acoustical interference for different ventilation hole designs

\begin{tabular}{|c|c|c|c|c|}
\hline $\mathrm{N}$ & $\begin{array}{c}\text { Diameter } \\
\mathrm{D} \\
(\mu \mathrm{m})\end{array}$ & $\begin{array}{c}\text { Measured } \\
\mathrm{f}_{\text {low }} \\
(\mathrm{Hz})\end{array}$ & $\begin{array}{c}\text { Theoretical } \\
\mathrm{f}_{\text {low }} \\
(\mathrm{Hz})\end{array}$ & $\begin{array}{c}\text { Max. SPL for 1dB } \\
\text { compression } \\
(\mathrm{dB} \mathrm{SPL})\end{array}$ \\
\hline 4 & 5 & $<2$ & 2 & 126 \\
\hline 4 & 10 & 20 & 24 & 127 \\
\hline 18 & 10 & 95 & 106 & 129 \\
\hline 36 & 10 & 190 & 211 & 133 \\
\hline
\end{tabular}

Environmental noise is typically characterized by dominating low frequency contributions. Therefore, a microphone with reduced sensitivity at low frequencies partially suppresses environmental noise such as wind noise, door slam or even tapping on the sound port.

Figure 10 summarizes the environmental noise suppression depending on the lower corner frequency. It was measured with an exemplary noise distribution originating from wind noises. The noise suppression is defined by the microphone noise voltage (A-weighted and non weighted in the bandwidth of $10 \mathrm{~Hz}$ to $10 \mathrm{kHz}$ ) in relation to the noise voltage of a microphone with a corner frequency of $<2 \mathrm{~Hz}$. For a microphone with a corner frequency of $190 \mathrm{~Hz}$, a wind noise suppression of approximately 5.1 $\mathrm{dB}$ can be achieved which gives additional head room for the amplifier to transmit a non clipping signal.

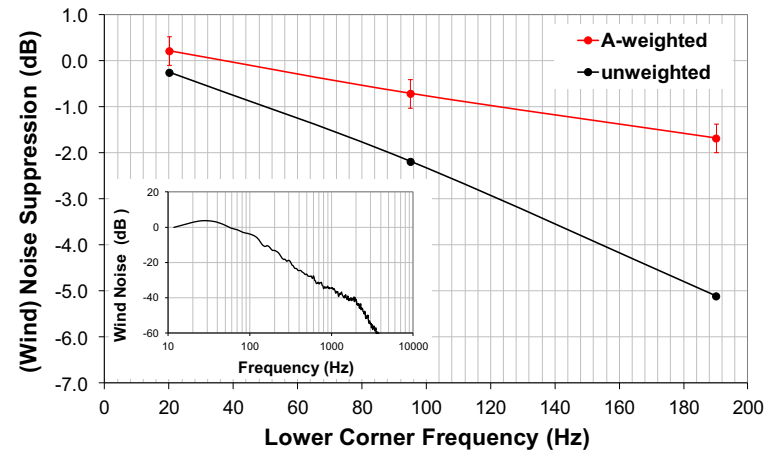

Fig. 10 Relative environmental noise suppression depending on the lower corner frequency for a typical wind noise test spectrum (small graph).

\section{Simulation and Measurement}

Acoustical measurements are done under free field conditions in the case of sensitivity. To measure noise accurately environmental signals must be sealed off well. Closing the sound port would cancel the noise of the sound port instead such measurements must be done in an anechoic chamber.

Figure 11 shows a sensitivity measurement of the large membrane microphone that clearly reveals the $3 \mathrm{~dB}$ high pass at $80 \mathrm{~Hz}$ and the resonance close to $22 \mathrm{kHz}$ originating from the air mass in the sound port and the microphone spring constant.

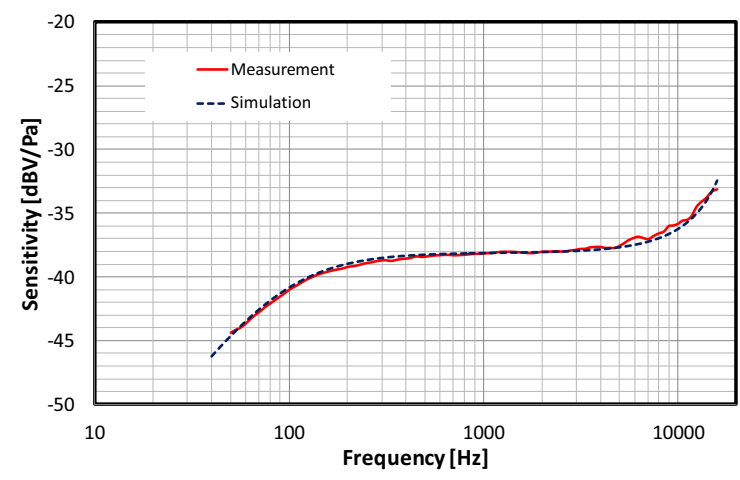

Fig. 11: Comparison of measurement versus simulation of frequency response of the $1.1 \mathrm{~mm}$ diameter membrane microphone.

The total noise of several microphone samples is displayed in figure 12 . The high quality of measurement can be seen from the absence of any distorting signal: neither $50 \mathrm{~Hz} / 100 \mathrm{~Hz}$ EMI nor body noise is visible. At low frequencies the spectral noise density drops with $12 \mathrm{~dB}$ per decade. At medium frequencies thermal noise is dominating and the sound port generates a significant contribution at about $22 \mathrm{kHz}$ due to the above mentioned resonance. This is outside the audio band and hence has negligible influence on total noise. 


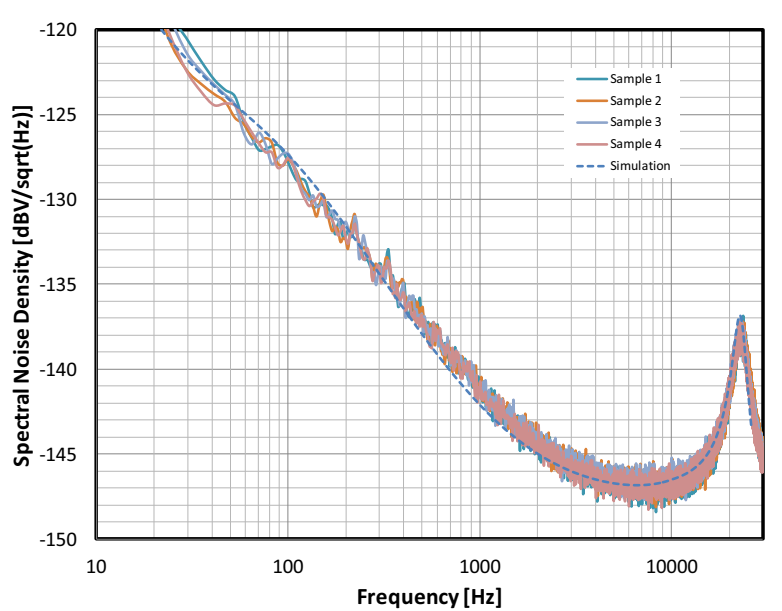

Fig. 12 Measurements and simulation of microphone system noise.

Since the noise spectral density is very well reflected by the small signal equivalent lumped element model a deeper analysis of noise sources is meaningful (tab. 3).

Tab. 3: Detailed measurement results compared with simulation.

\begin{tabular}{|r|c|c|c|c|c|c|c|}
\hline & & $\mathrm{S} 1$ & $\mathrm{~S} 2$ & $\mathrm{~S} 3$ & $\mathrm{~S} 4$ & Average & Simulation \\
\hline $\mathrm{S} 1 \mathrm{kHz}$ & $\mathrm{dBV} / \mathrm{Pa}$ & -37.7 & -37.8 & -37.9 & -38.0 & -37.8 & -37.7 \\
\hline $\mathrm{SNR}$ & $\mathrm{dB}(\mathrm{A})$ & 66.4 & 66.1 & 65.8 & 66.0 & 66.1 & 66.2 \\
\hline $\mathrm{f}_{\mathrm{low}}$ & $\mathrm{Hz}$ & 91 & 91 & 92 & 92 & 91.7 & 92.7 \\
\hline
\end{tabular}

According to the detailed simulation in figure 13 the total noise is well balanced between the MEMS transducer $(53 \%)$ and the ASIC $(47 \%)$. The electronic noise has a significant $1 / \mathrm{f}$ low frequency contribution typical for the PMOS source follower which is the first amplifier stage in the ASIC. The package sound port has a minor contribution of $3 \%$. The MEMS transducer has two contributions. The thermal noise of the ventilation hole is filtered by the back volume compliance and shows the drop over frequency adding up to a $16 \%$ contribution. The largest noise adder is related to the design of the backplate. Fluidic damping through the perforation and in the air gap has a broad band effect of $34 \%$. From fluidic simulation we know that the perforation density and perforation hole diameter are the relevant parameter. In the given case $8 \mu \mathrm{m}$ diameter holes are arranged with a density of $69 \%$. Higher densities or hole diameters are difficult to achieve without signal loss due to reduced capacitance of the transducer.

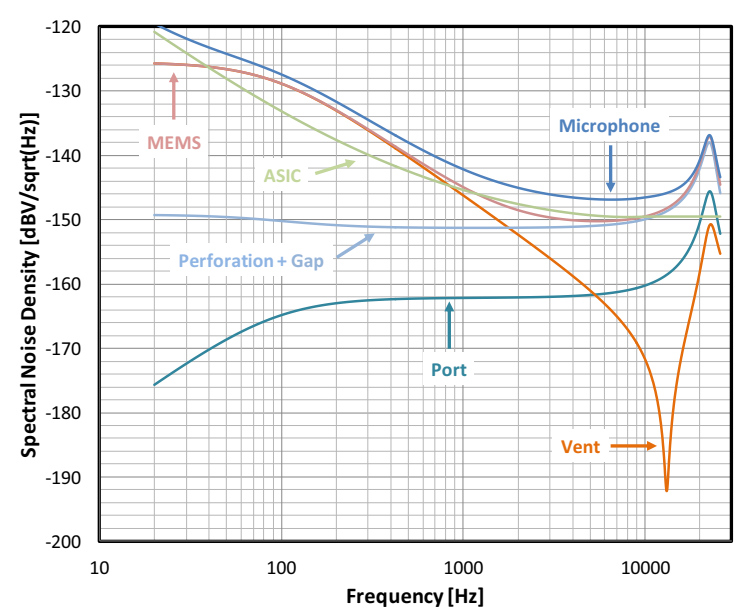

Fig. 13 Simulation of spectral noise density of microphone module and the specific noise contributors.

\section{Conclusion}

Silicon MEMS technology has enabled a technology change from can type electret microphones to surface mountable and reflow solderable microphones. Moreover, performances in access of $66 \mathrm{~dB}$ SNR are state of the art with package height below $1 \mathrm{~mm}$. Further optimization and scaling will enable high end applications with form factors of several $\mathrm{mm}^{3}$. Applications are already heading to double the signal to noise ratio towards $72 \mathrm{~dB}(\mathrm{~A})$.

\section{References}

[1] A. Dehé, Aigner, T. Bever, K.G. Oppermann, E. Pettenpaul, S. Schmitt and H.-J. Timme, Silicon micromachined microphone chip at Siemens, $137^{\text {th }}$ Meeting of the Acoustical Society of America and $2^{\text {nd }}$ Convention of the European Acoustic Association, Berlin, March 14-19, 1999; Acta Acustica, Vol. 85, 1999

[2] A. Dehé, M. Füldner, J.-P. Sommer, K.-F. Becker, M. Koch. Silicon Microphones for Automotive and Hearing Aids, in The World of Electronic Packaging and System Integration,ddp Goldenbogen, Dresden 2004, pp 278-84.

[3] M. Füldner, A. Dehé and R. Lerch, Analytical Analysis and Finite Element Simulation of Advanced Membranes for Silicon Microphones, IEEE Sensors Journal, October 2005, vol. 5, no. 5, pp. 857-63.

[4] R. Sattler, Physikalisch basierte MixedLevel Modellierung von gedämpften elektromechanischen Mikrosystemen, PHD thesis, Munich, Nov. 2007 